\title{
Editorial
}

\section{Darwin, Evolution and the Origin of Species}

\author{
Ram B. Singh ${ }^{1, *}$ and Favien De Meester ${ }^{2}$
}

\section{${ }^{1}$ Halberg Hospital and Research Institute, Moradabad, India, ${ }^{2}$ Columbus Paradigm Institute, Waterloo, Belgium}

February 12th is the 200th anniversary of Darwin's birth. After 127 years of his death, researchers are still grappling with some details of the theory of evolution. However, Darwin only briefly addressed how quickly evolution might occur.

On August 29, 1831, Charles Darwin returned home from a geology field trip in North Wales. He got a letter waiting for him from his Cambridge professor and mentor, John Stevens Henslow. It contained an invitation to voyage around the world as a naturalist on the HMS Beagle, scheduled to leave a month later. Darwin's father strongly opposed the idea, saying the plan was rushed, reckless, and detrimental to Darwin Jr.'s career prospects as a clergyman. The next day, Darwin who was 22 years old, rode some 50 kilometers from his home in Shrewsbury to Maer Hall to visit his uncle Josiah Wedgwood. Darwin relayed his father's objections and pleaded his case. Darwin conceived the first glimmering of his theory of evolution on that particular voyage, which he described in "On the Origin of Species", published 150 years ago (February 12 is also the 200th anniversary of Darwin's birth). It seems that if he had not gone on the voyage, it may had posed a real problem for him. It may have caused bitterness in relations and that might have disrupted him in pursuing his studies.

In The Origin of Species, Darwin wrote: "Natural selection can act only by taking advantage of slight successive variations; it can never take a leap, but must advance by the shortest and slowest steps". It is possible, he believed that organisms gradually adapt to their environments via minute physical adjustments. Biologists have since found evidence that dramatic adaptations can also occur, for instance in the form of major morphological changes. These adaptations appear to be the cause of changes in structure from apes to man and various human races. It is not clear whether such adaptations may be responsible for the development of diseases or of good health [1-4].

The history of evolution of human beings indicate that our early diet included natural foods; fruits,vegetables, green vegetables, seeds, eggs and honey. Fish, and lean meat were also available to apes as well as to early humans which shaped modern human's genetic nutritional requirement. Since the emergence of Homo erectus 1.7 million years ago, the apes have transformed in to Homo sapien men without much change in the nutrient content of diet [5]. During the last 10,000 years we had $<500$ generations which were without maladaptation and mutations. Our genes appear to be similar to the genes of our ancestors during the Paleolithic period 40,000 years ago, at which time our genetic profile was established. However, this could be due to a better adaptation. The evolution of obesity, central obesity, cardiovascular diseases (CVD), diabetes, hypertension and cancer may occur due to maladaptations against environmental factors [1-4]. The adaptations could be molecular; genetic, immunological, hormonal and morphological in the form of obesity and other changes. These diseases are polygenic in nature and their prevalences and mortality vary depending upon genetic susceptibility and presence of risk $[1,2]$. There is an evidence regarding the role of nutritional and genetic variance in the occurrence of these chronic diseases. The genetic variance in cancer appears to have greater genetic component as compared to coronary artery disease (CAD), hypertension, obesity, and diabetes. In primary or secondary prevention of chronic diseases, every effort should be made to prevent the expressions of genotype resulting into phenotype risk factor, by treating the cause which may be from the environment.

The preventive efforts should begin in pregnancy and infancy because during this stage there is greater molecular susceptibility of the subject to environmental factors. There is also an evidence that the lipoprotein (a) phenotype can change during childhood and possibly also during pregnancy. Lack of energy and iron during pregnancy may cause development of conserving mechanisms, due to adaptations, in the mother and the fetus, which may be harmful during infancy, on modest increase in these nutrients. While iron conservation may increase free radical generation, and can damage the genes, energy conservation may result in to obesity and central obesity, on modest intake in food intakes, due to interaction of gene and environmental factors. It is also possible that time frame may also influence the functioning of genes.

The theory of complexity figures out the relative speed with which organisms of varying complexity might be able to adapt and evolve. Complexity correlates with the number of specially-adapted traits, estimating the speed of evolution even in simple organisms. Viruses can evolve in a matter of generations, but, as always, the strength of the selective pressure in an environment affects how fast organisms adapt, so individuals of the same species in different environments will evolve at different rates. The key assumption is that a single mutation can potentially alter any and all traits in an organism, including those that are advantageous. This concept, that a mutation can affect most traits is referred to as universal pleiotropy. However, it is 
nuclear how extensive pleiotropy really is. If mutations affect just a small percentage of traits, rather than the majority, then complexity would act as less of a speed bump for evolutionary change. There is a need to gather data on how common pleiotropy is, and what percent of characters in an organism are affected by random mutations.

In early 1990's, experiments carried out on Drosophila reveal that two-thirds of all the genes are involved in correct development and neural connectivity of the eye. Therefore, mutations, even in indirectly related genes, can affect a broad range of traits. There is another evidence that there is a lot of pleiotropy out there; it is unclear, how many characters are affected. Pleiotropy acts as a speed limit on evolution in some capacity, but whether it's a strong limit, or weak one, is still unanswered. Pleiotropy may be extensive, but not paralyzing. Evolution is neither infinitely nor negligibly flexible, creating an intermediate state where the ability of an organism, whether simple or complex, to evolve is kept in moderate check by the effect of mutations. These mutations could be spontaneous or may occur due to interaction of environmental factors, diet, pollutants, radiations, sun light, which act by causing free radical stress [4]. Free radicals are damaging to genes which can cause mutations and enhance genetic expressions. However, internal environment of an individual may be protected by the presence of antioxidants, vitamins and minerals which may be helpful in adaptations. These findings suggest that questions of how fast a particular species, whether complex or not, evolves will be complicated by the fact that, within species, some individuals will have an easier time adapting to their changing environment. Therefore, in mouse strains there may be a genetic lineage better suited for making most of the mutations that pop up, and more quickly adapting to their environments-a factor that cannot be accounted for the experiments. The ability to adapt to a new environment can differ among genotypes and among species. Evolution itself has the ability to evolve, but genotype is also important, which depends on the interaction with environmental factors. The internal nutrient environment as well as external environmental factors can influence adaptations and evolution of various characters in a specie or individual, which may result in to disease.

\section{REFERENCES}

[1] Singh RB, Mori H. Nutritional adaptation and risk factors of coronary heart disease. J Nutr Med 1992; 3: 311-8.

[2] Singh RB, Mori H. Risk factors for coronary heart disease, synthesis of a new hypothesis through adaptation. Med Hypotheses 1992; 39: 334-41.

[3] Singh RB, Niaz MA. Genetic variation and nutrition, in relation to coronary artery disease. J Assoc Phys India 1999; 47: 1185-90.

[4] Houstis N, Rosen ED, Lander ES. Reactive oxygen species have a causal role in multiple forms of insulin resistance. Nature 2006; 440: $944-8$.

[5] Eaton SB, Conner M. Paleolithic nutrition: a consideration of its nature and current implications. N Engl J Med 1985; 312 : 283-9. 